\title{
Design of a Short Tensile Coupon for Fiber Reinforced Plastic Using Artificial Neural Networks
}

\author{
Mehmet Saribiyik ${ }^{1, *}$, Naci Caglar ${ }^{2}$, and Seyhan Firat ${ }^{1}$ \\ 'Sakarya University, Department of Construction, 54187 Adapazari, Turkey \\ ${ }^{2}$ Sakarya University, Department of Civil Engineering, 54187 Adapazari, Turkey
}

\begin{abstract}
The measurement of the mechanical properties of Fibre Reinforced Plastic (FRP) material is necessary for numerical structural analysis and design. The mechanical properties of the FRP materials may be determined by specific coupon test methods or by analytical calculation. However, pultruded or moulded FRP components may not possess the dimensions to permit the extraction of standard length coupons. The shape of the short tensile coupon has been established to circumvent this limitation using a Finite Element (FE) representation and Artificial Neural Network (ANN) including the effect of gripping length, coupon shape, width, length and thickness. The FE results have been used for the learning and testing sets of the ANN. The Multi-Layer Perceptron (MLP) has been employed in the modelling of the ANN. The MLP model has been trained using the Scaled Conjugate Gradient Algorithm (SCGA) and tested. The ANN results show that the correlation between targets and outputs are consistent.
\end{abstract}

Key words: Fibre reinforced plastic, mechanical properties, numerical analysis, tensile coupon, artificial neural network, scaled conjugate gradient algorithm.

\section{INTRODUCTION}

There has been a rapid increase in the development of advanced composites incorporating fine fibres, termed Fibre Reinforced Plastic (FRP). The FRP structural members are currently produced successfully by pultrusion and are used in a number of civil engineering applications. The pultrusion process has enabled the FRP to become a competitive alternative to traditional structural materials (steel, concrete and wood, etc). It also offers advantages over conventional materials such as a lower specific weight with respect to strength, good environmental durability, versatility in fabrication process and provides electrical and flame resistance subject to the materials selected. Material surfaces are also durable and require little maintenance.

Assuming a state of plane stress, measurement of the mechanical properties of highly orthotropic material is necessary for numerical structural analysis and design. A fundamental assumption used, when determining the elastic properties of the material from a tensile test, is the existence of a uniform state of strain within the gauge length. The gripping action of the jaws, in applying the axial load, restrains the coupon displacement in the transverse direction such that the corresponding strain, $\varepsilon_{y}$, is similarly restrained. For a short coupon, the effects are caused the strain distribution at mid-length (in the vicinity of the gauge) to become non-uniform. Consequently, $\varepsilon_{x}=\sigma / E_{x}$ is no longer valid as the state of strain at the centre of the coupon and is not consistent with the assumed value of stress.

Classically, the undesirable constraining effects of the jaws have been minimised by providing an adequate coupon length. However, the geometry of many structural FRP sections prevents the extraction of a

\footnotetext{
' Corresponding Author, Tel: +90-26434602 60,

Fax:+90-264 3460262, E-mail: mehmets@sakarya.edu.tr
} 
standard length specimen (typically in excess of 250 $\mathrm{mm}$ ) for the evaluation of elastic properties in the transverse direction as defined by the most commonly used standards (BS 2782, ASTM D638 and ASTM D3039) ${ }^{\#}$. Despite the use of non-standard tests and outcomes $/ 1-2 /$, the provision of the specification of a short coupon impose a requirement to ensure the determination of reliable material properties.

Finite element method has been effectively used to establish test specimen characteristics and loading configurations for composite materials. Reported researchers /3-7/ have concentrated on the improvement of standard length coupons in aiming to reduce stress concentrations at the clamping mechanism leading to an underestimate of tensile strength of the test material. The off-axis techniques for the tension specimens are good example of the application of numerical methods 13/. The influence of tabs on the indicated strength of the FRP specimens and the level of gripping were investigated using a linear-finite-element method. The results indicate that it is possible to produce nearly uniform states of stress in the gauge length with the proposed tab and clamping configurations. Sandhu and Sendeckyj /4/ determined that a state of uniform axial stress within the test section could be achieved if the grips were allowed to rotate and the fibre angle of the parent tab material and tab geometries were simultaneously optimised. Kural and Flaggs /5/ used the results of two-dimensional plane stress finite element analyses to improve the design by achieving increased uniformity of the strain and minimisation of the stress concentrations within a tensile coupon near the tabs. Foos et al. /6/ used two-dimensional linear finite element analyses for the design of the end tabs to improve the uniformity of the strain state in a tensile test specimen in order to obtain an accurate measurement of the tensile strength of graphite/epoxy composites. It was demonstrated that the uniformity of the strain states within the tensile test specimens could be improved by

\footnotetext{
"BS 2782: "Plastic - Determination of Tensile Properties".
}

ASTM D638: "Standard Test Method for Tensile Properties of Plastics".

ASTM D3039: "Standard Test Method for Tensile Properties of Polymeric Composite Materials". modifying the coupon design using the results of finite element analysis. Linear elastic analysis was also found to be adequate in this case. Gosling and Saribiyik /7/ studied to establish the shape of a short tensile test coupon under a length constraint using the finite element method to determine the elastic properties of the FRP materials.

It appears to offer a means of dealing with many multi-variety problems for which an exact analytical model does not exist or at least is very difficult and time consuming to develop the solution. Therefore, it is necessary to use a new algorithm to solve the mentioned time consuming problems and difficulties. In recent years, Artificial Neural Network (ANN) has been effectively applied in many engineering applications, such as pattern recognition $18,9 /$, image and signal processing $/ 10 /$, and structural optimization problems $111,12 /$. The ANN has also been applied in several areas of computational and experimental mechanics /13, $14,15 /$, earthquake engineering $/ 16,17 /$, and structural engineering /18/. Lee at al. $/ 19 /$ investigated the use of ANN for the prediction of fatigue lives of composite materials. It was found that the ANN can be trained to model constant-stress fatigue behaviour at least as well as other current life-prediction methods and can provide accurate representation of the stress/R-ratio/median-life surface for carbon fibre composites. Kasperkiewics /20/ applied the ANN in certain materials-analysis problems and suggested to use for predicting properties in the simulation of concrete-like composite materials in design and optimisation. The ANN was trained successfully how to predict the ultimate shear force in concrete beams from known data concerning six input parameters: the geometrical characteristics for the beam depth, width and length; the percentages of the horizontal and vertical web reinforcement; and the value of the compressive strength of the concrete 121 . Recently, the acoustic emissions, which are a highly promising technique for evaluation of failure modes in composite materials, were classified using the ANN. The results demonstrated that the damage characterization in glass/epoxy composite for different stacking sequences can be done using ANN automatically $/ 22 \%$. 
The objective of this study is to investigate the applicability of the ANN as an alternative method to propose the shape of a short tensile coupon for the determination of both elastic modulus and strength of the FRP. Learning and testing sets of the ANN models have been developed using the FE results, which predict strain fields at the centre of coupons under length constraints.

\section{ARTIFICIAL NEURAL NETWORK}

The ANN is a powerful data modelling tool that is able to capture and represent complex relationships between inputs and outputs. Its instants are composed of large numbers of highly interconnected processing elements that are called as neurons and are tied together with weighted connections. Each neuron works as an independent processing element, and has an associated transfer function, which describes how the weighted sum of its inputs is converted to the results into an output value. Each hidden or output neuron receives a number of weighted input signals from each of the units of the preceding layer and generates only one output value (Fig. 1).

The most basic and commonly used ANN is a MultiLayer Perceptron (MLP). It consists of at least three or more layers, which comprises an input layer, an output layer and a number of hidden layers. Each neuron in one layer is connected to the neurons in the next layer and there are no connections within a single layer. The number of neurons in each layer may vary, depending on the problem (Fig. 2).

The weighted sum of input components is calculated as:

$$
N e t_{j}=\sum_{i=1}^{n} w_{i j} x_{i}
$$

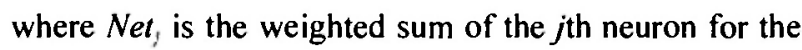
input received from the preceding layer with $\mathrm{n}$ neurons, $w_{i j}$ is the weight between the $j$ th neuron and the $i$ th neuron in the preceding layer, $x_{1}$ is the output of the $i$ th neuron in the preceding layer. The output of the $j$ th neuron out, is calculated with a sigmoid function as follows:

$$
o u t_{j}=f\left(N e t_{j}\right)=\frac{1}{1+\exp \left(-k N e t_{j}\right)}
$$

where, $k$ is a constant used to control the slope of the semi-linear region. The sigmoid nonlinearity activates in every layer except the input layer $/ 14 /$.

Back Propagation Algorithm (BPA), which is one of the most famous training algorithms for the MLP $/ 21$, is a gradient descent technique to minimise the error for a particular training pattern. BPA is used to adjust the weights a small amount at a time in a way that reduces the error. In the Conjugate Gradient Algorithm (CGA), a search is performied along conjugate directions, which produces generally faster convergence than steepest descent directions /11/. A Scaled Conjugate Gradient Algorithm (SCGA) is designed to avoid the timeconsuming line search in each iteration, because it is computationally expensive in CGA. The SCGA combines the model-trust region approach, which is used in the Levenberg-Marquardt algorithm with the CGA. Detailed description can be found in reference /23/.

The training of the network is accomplished by adjusting the weights and is carried out through a large number of training sets and training cycles (epochs). The goal of the learning procedure is to find the optimal set of weights, which in the ideal case would produce the right output for any input. The output of the network is compared with a desired response to produce an error. The performance of the MLP is measured in terms of a desired signal and the criterion for convergence. It is determined by the root mean square error, which is expressed as:

$$
\varepsilon_{R M S}=\sqrt{\frac{\sum_{i=1}^{m}\left(T_{i}-o u t_{i}\right)^{2}}{m}}
$$

where $T_{1}$ and out $_{1}$ are the desired (target) and actual output values respectively for ith output neuron, and $m$ is the number of neurons in the output layer. Once the ANN is adequately trained, it can generalize to similar cases, which it has never seen. 


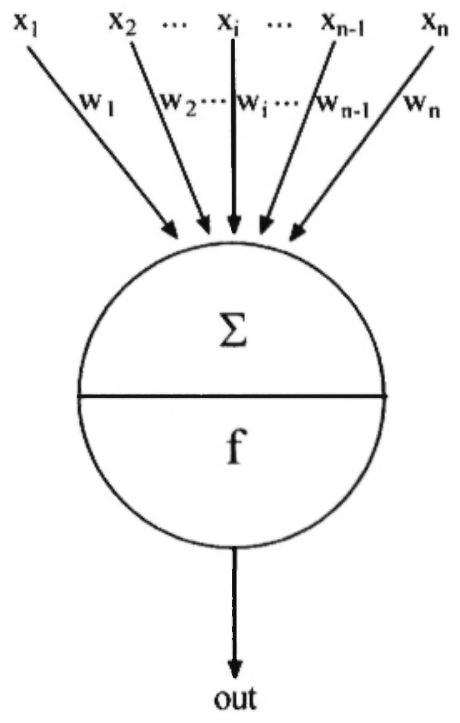

Fig. 1: The structure of an artificial neuron.

\section{COUPON GEOMETRIES - FINITE ELEMENT ANALYSIS}

Numerical models of proposed test coupon geometries have been prepared and analysed using the finite element system, LUSAS $17 \%$. A typical numerical model of a coupon consists of two types of isoparametric elements. Both element types feature quadratic shape functions and are capable of modelling non-rectangular shapes and curved boundaries in the plane, consistent with potential coupon geometries.

The main configurations of the short coupon and ASTM D3039 coupon models are shown in Figs. 3 and 4. An applied uniform shear stress (surfaces $S I-S 4$ ) has been used to represent the application of the axial load to the coupon (surfaces S/-S4, Fig. 3). The physical support conditions have been introduced through the following displacement constraint equations:

$$
\begin{aligned}
& u \neq 0, v=0, w=0, \quad(\text { surfaces } S /-S 4) \\
& u=0, v \neq 0, w \neq 0, \quad(\text { surface } S 5)
\end{aligned}
$$

Constraint (Eq. 4) admits longitudinal displacement but prevents the Poisson's ratio effect in the $y$-direction, consistent with the gripping effect of the jaws. Eq. (5) effectively prevents the numerical representation from

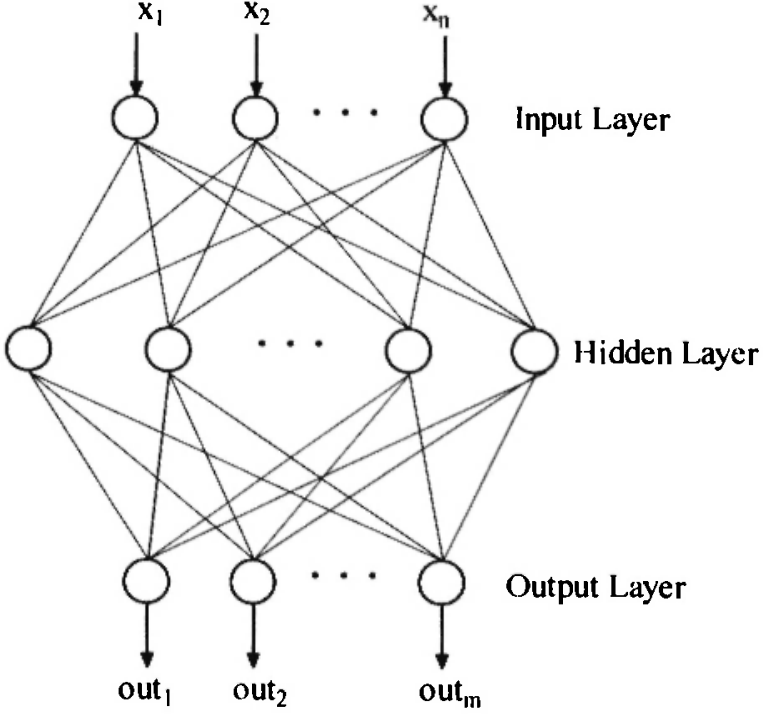

Fig. 2: A typical multi-layer perceptron neural network.

being poorly conditioned as a result of a potential rigidbody translation in the global $x$ direction and rigid body rotation about the global $z$-axis.

\subsection{ASTM D3039 Model}

The estimated properties of a FRP material depend on the fibre properties, matrix properties, fibre volume fraction, and the architecture of the fibres in the lamina. The fibre volume fraction of each ply is defined as the ratio of the volume of fibres present in a layer to the total volume of that layer. The mechanical properties of the fibre and the matrix are used to calculate elastic properties of each layer using micro-mechanics. The outcomes of these calculations are then used in a macromechanical model to estimate the material properties of the FRP box section through classical lamination theory to establish the validity of the numerical formulation $/ 7$, $24 /$.

Assuming linear elasticity and a prismatic section, the longitudinal strain in the central region of the specimen, $E_{r}$, is:

$$
\varepsilon_{r}=\frac{P}{E_{x} A}
$$




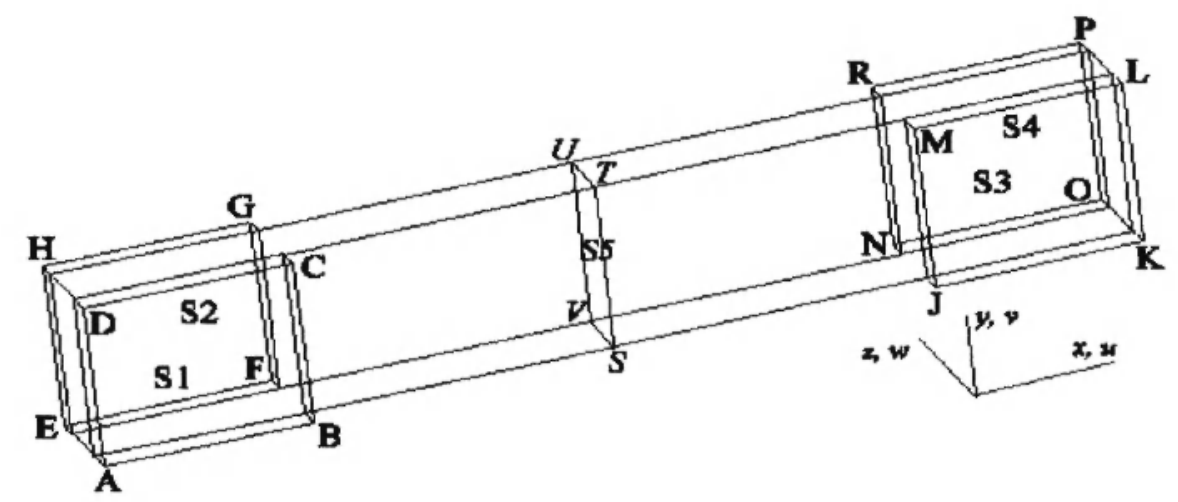

Fig. 3: Schematic of coupon model.

$(\mathrm{S} 1 \rightarrow \mathrm{ABCD} ; \mathrm{S} 2 \rightarrow \mathrm{EFGH} ; \mathrm{S} 3 \rightarrow \mathrm{JKLM} ; \quad \mathrm{S} 4 \rightarrow \mathrm{NOPR} ; \mathrm{S} 5 \rightarrow \mathrm{STUV})$.

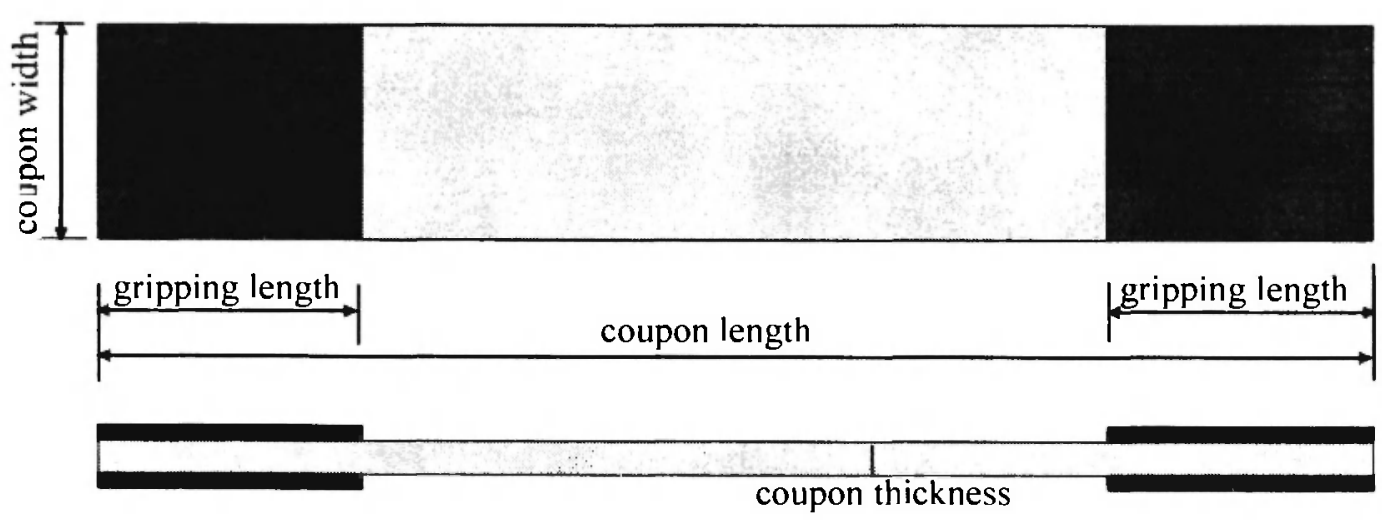

Fig. 4: Schematic of ASTM coupon model.

where $P$ is axially applied load, $E_{x}$ is elastic modulus in longitudinal direction $\left(27.36 \mathrm{kN} / \mathrm{mm}^{2}\right)$ and $A$ is the cross-sectional area of the specimen $(3.1 \mathrm{~mm} \times 15 \mathrm{~mm}$ $=46.5 \mathrm{~mm}^{2}$ ) (Fig. 5). The analysis is linear elastic. Therefore, an arbitrary value of $P$ may be assumed within the elastic range and ultimate strength of the material, $15 \mathrm{kN}=323 \mathrm{kN} / \mathrm{mm}^{2}<\sigma_{m i t}=388 \mathrm{kN} / \mathrm{mm}^{2}$ ) $124 /$.

The magnitudes of the analytical strain $\left(\varepsilon_{x}=\right.$ 0.01179 ) and Gauss point results obtained from elements located at the centre of the "numerical coupon" coincide subjected to each other. The computed strain distribution is shown in Fig. 6 for the entire coupon. The non-linearity (with length) of the axial strain beyond the extent of the tabs is clearly visible and is seen to dissipate rapidly towards to the centre of the coupon. Using this interval to display strain variations at the centre of the ASTM D3039 specimen would lead to no contours being displayed implying a strain variation of less than $0.6 \times 10 \mathrm{E}-04$ in the gauge area. The equality of the analytical and computed strains at the centre demonstrates the validity of the numerical model, while the philosophy behind a minimum length is indicated by the strain smoothing away from the end restraints. A comparison with experimental data is not necessary here because the quality of the strain distribution (i.e. uniformity) is to be measured against an analytical target.

\subsection{Variations of Short Coupon Model}

Adopting the numerical modelling described in the previous section, short coupons with a fixed length of $50 \mathrm{~mm}$, a thickness of $3.1 \mathrm{~mm}$ and a gripping length of 


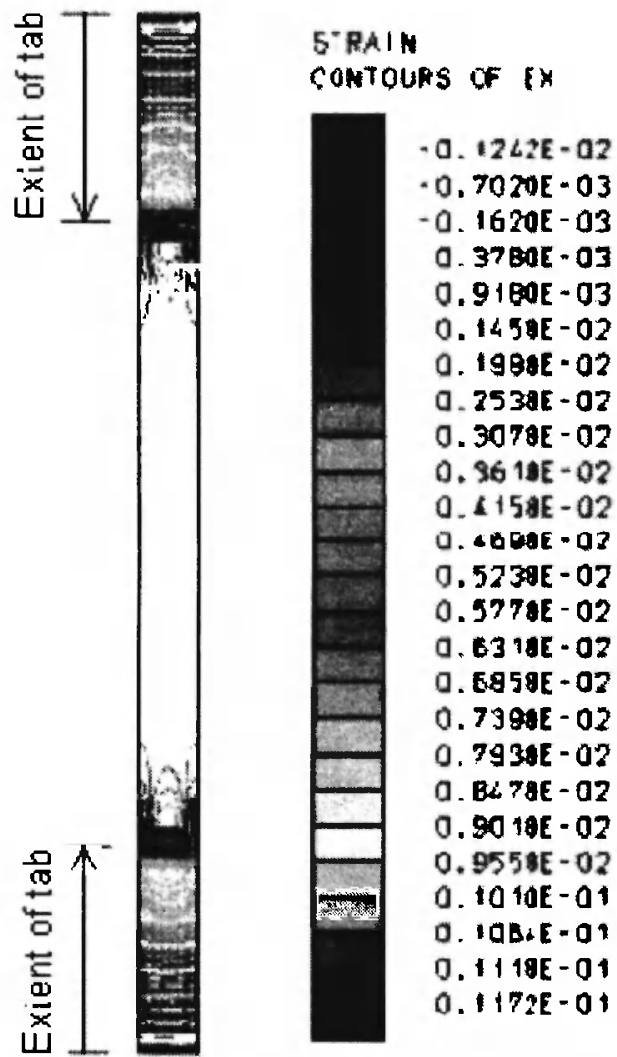

Fig. 5: Strain contours for ASTM D3039 specimens in the longitudinal direction.

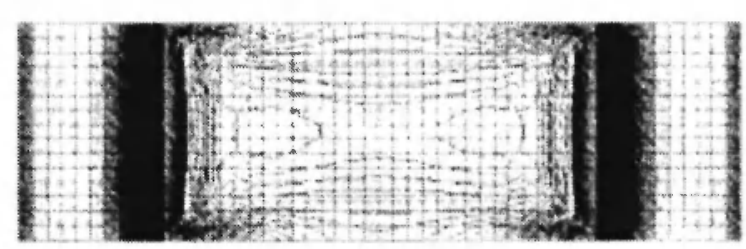

(a)

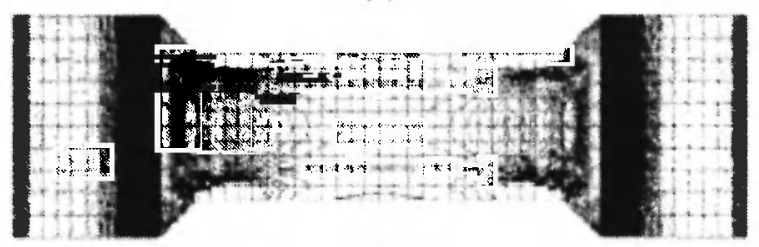

(b)

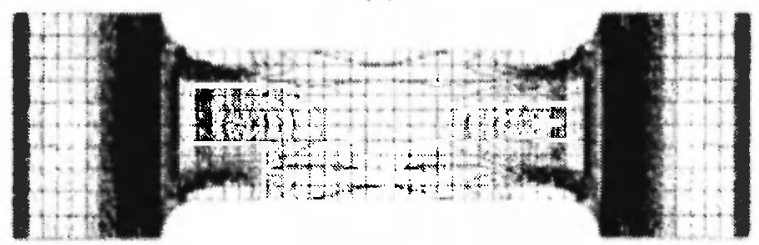

(c)

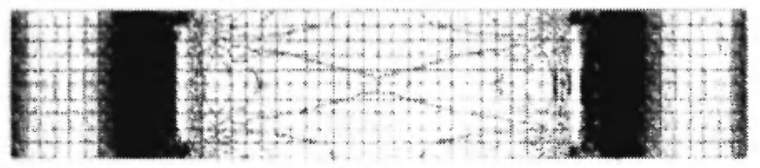

(d)

Fig. 6: Contours of longitudinal strain $-50 \mathrm{~mm}$ short coupons.

Table 1

Coupon specifications for ANN model.

\begin{tabular}{|c|c|c|c|c|}
\hline $\begin{array}{c}\text { Coupon Shape } \\
(\mathbf{m m})\end{array}$ & $\begin{array}{c}\text { Coupon Width } \\
\text { (mm) }\end{array}$ & $\begin{array}{c}\text { Gripping length } \\
\text { (mm) }\end{array}$ & $\begin{array}{c}\text { Coupon Length } \\
\text { (mm) }\end{array}$ & $\begin{array}{c}\text { Coupon thickness } \\
\text { (mm) }\end{array}$ \\
\hline \multirow{4}{*}{$50-10$ st } & 5 to 20 & 10 & 50 & 3.1 \\
\cline { 2 - 5 } & 10 & 10 & 30 to 90 & 3.1 \\
\cline { 2 - 5 } & 10 & 6 to 14 & 50 & 3.1 \\
\cline { 2 - 5 } & 10 & 10 & 50 & 2 to 6 \\
\cline { 2 - 5 } & 10 & 10 & 40 & 2 to 6 \\
\hline
\end{tabular}


$10 \mathrm{~mm}$ and two generic shapes have been analysed. A short version of the ASTM straight-sided coupon with $10 \mathrm{~mm}$ and $15 \mathrm{~mm}$ widths has been used to represent the first type. For the second type, the effects of strategies used to reduce the gauge width beside the gripping area on the central strain distribution has been investigated, especially in the context of promoting failure within the gauge area and away from the clamping zones. Two approaches have been used to develop the reduced width transition. The first is a simple $45^{\circ}$ gradient (see Fig. 6(b)) and the second an arc with a radius of $2.5 \mathrm{~mm}$ and the origin chosen to generate a quarter circle (see Fig. 6(c)). Simple geometries have been proposed in order to assist in the fabrication of the coupons.

With a mesh of $1 \mathrm{~mm}$ by $1 \mathrm{~mm}$ squares plotted on the surfaces of the coupons (Fig. 6), the quality of the strain distribution may be judged by the number of contours in the context of a typical strain rosette with plan dimensions $9 \mathrm{~mm}$ wide by $6 \mathrm{~mm}$ long (i.e. EA-13060RZ-120). A comparison of longitudinal strain distributions at the centre of the numerical models indicate that a coupon with a width transition introduced as an arc (denoted 50-2.5) performs well in comparison with the $15 \mathrm{~mm}$ straight-sided geometry (denoted 5015st) (Fig. 7). However, reducing the gauge width beyond the gripping area using an arc leads to high stress concentrations $(0.5028 / 0.3226=1.56)$ with the strain needing to "flow" into the reduced section. This stress concentration (important in the determination of the material tensile strength) is higher in the case of the $45^{\circ}$ linear transition example (denoted 50-45, see Fig. $6(b))$ with a corresponding ratio of $(0.6023 / 0.3226=$ 1.87). The $10 \mathrm{~mm}$ wide straight-sided performed well in comparison with a width transition introduced by a curve with a $2.5 \mathrm{~mm}$ radius (Fig. 6(c)). The same strain contour interval of $0.6 \times 10^{-4}$ is used for all diagrams, enabling a direct comparison of the uniformities of the strain field of each coupon type. The strain contour plots infer a number of performance characteristics of the coupon geometries. For example, in comparing the 15 $\mathrm{mm}$ and $10 \mathrm{~mm}$ wide coupon results (Fig. 6(a) and (d) respectively) it is clear that the strain field of one coupon cannot be interpolated. Furthermore, the rate of change of strain in the longitudinal direction is increased in the coupons involving a transition in width, with the effect exacerbated in the transverse direction at the same location, with negative implications in the accurate determination of the material length.

\section{COUPON GEOMETRIES - ANN APPLICATION}

The FE method has been used to evaluate the effects including the coupon shape, width, length, thickness and gripping length, one by one. To avoid the time consuming procedure the applicability of the ANN has been applied for the shape of the short tensile coupon including all effects (ie. coupon width, coupon length, coupon thickness and gripping length). Due to the lack of experimental results, training and testing sets for the ANN has been constituted using the FE analysis. From the outcomes of the FE study, the recommended straight sided short coupon (see Fig. 4), selected by comparing strain variations across the centre of the gauge area and stress concentrations, have been adopted for the ANN model. The numbers of nodes in input and output layers are based on the geometry of problem. But, the numbers of nodes in hidden layers and the numbers of the hidden layers are determined, generally, by trial and error. Therefore, the ANN model is selected as five nodes in input layer (Table 1), eight nodes in hidden layer and twenty nodes in output layer to define strain distributions at the centre of the short coupon (Fig. 8). In the ANN approach, type of backpropagation is SCGA, activation function is Sigmoidal Function, and number of epochs is 30000 . In order to test the ability of the proposed ANN based approach, the strain distributions have been compared with the FE results. The performance of the ANN showed that the correlations between targets and outputs are consistent as shown in Figs. 9 and 10.

\section{RESULTS AND DISCUSSION}

The FE results and the ANN based approach of four different outcomes, as defined in Figs. 11-14, have presented the applicability of the neural network based approach to propose the short tensile coupon. 


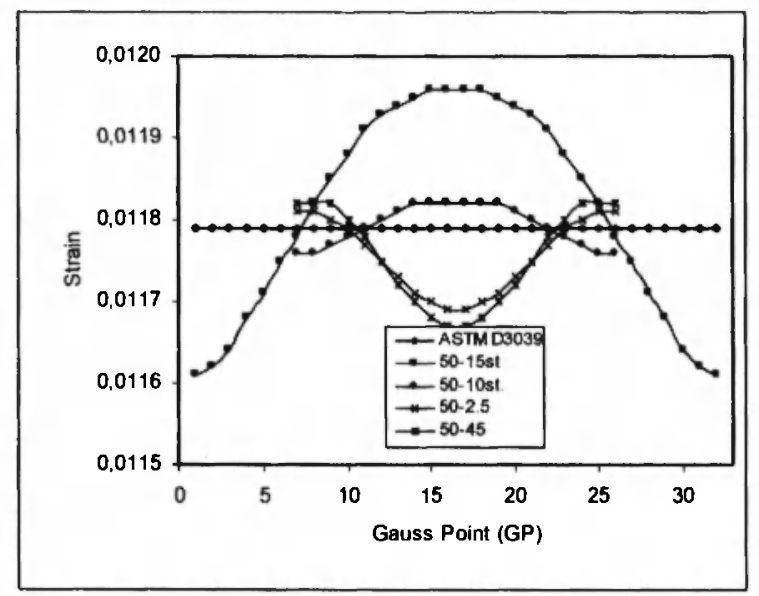

Fig. 7: Influence of coupon geometry on longitudinal strain distributions. (10 mm gripping length; $50-15 \mathrm{st}=15 \mathrm{~mm}$ rectangular, $50-10 \mathrm{st}=10 \mathrm{~mm}$ rectangular, $50-2.5=2.5 \mathrm{~mm}$ radius transition, $50-10-45=45^{\circ}$ linear transition).

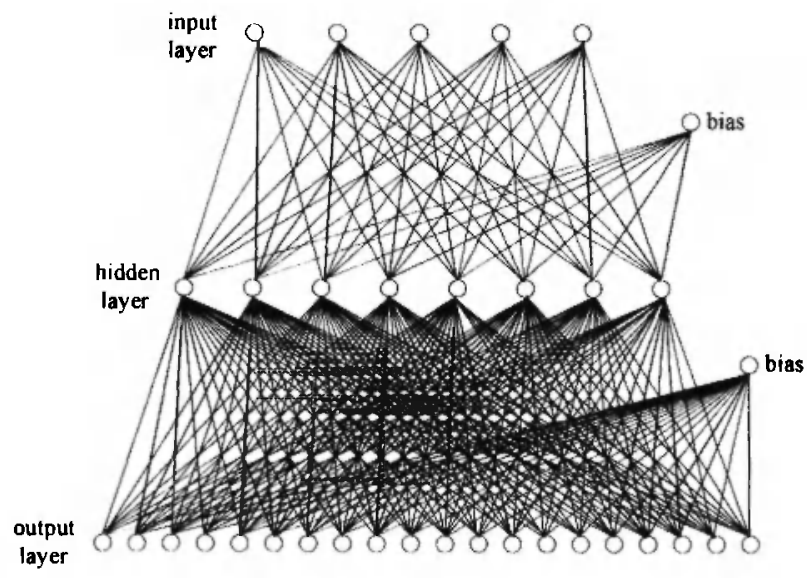

Fig. 8: Architecture of the ANN model.

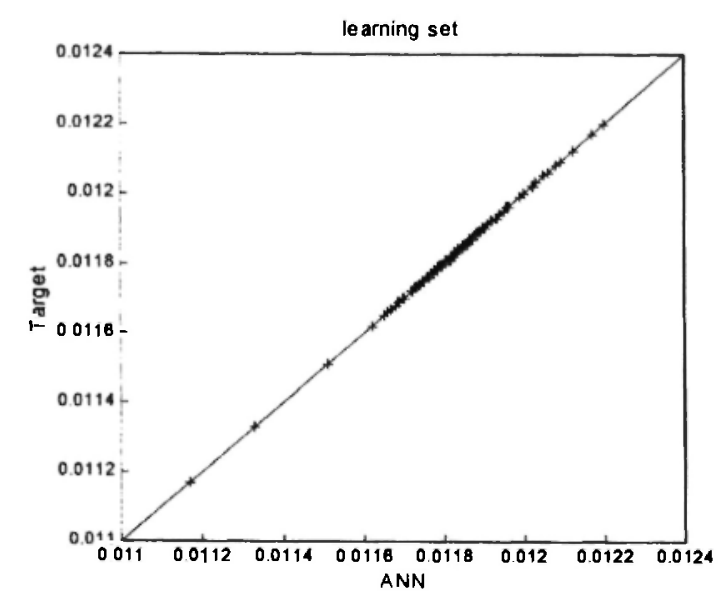

Fig. 9: Comparison of results for learning set

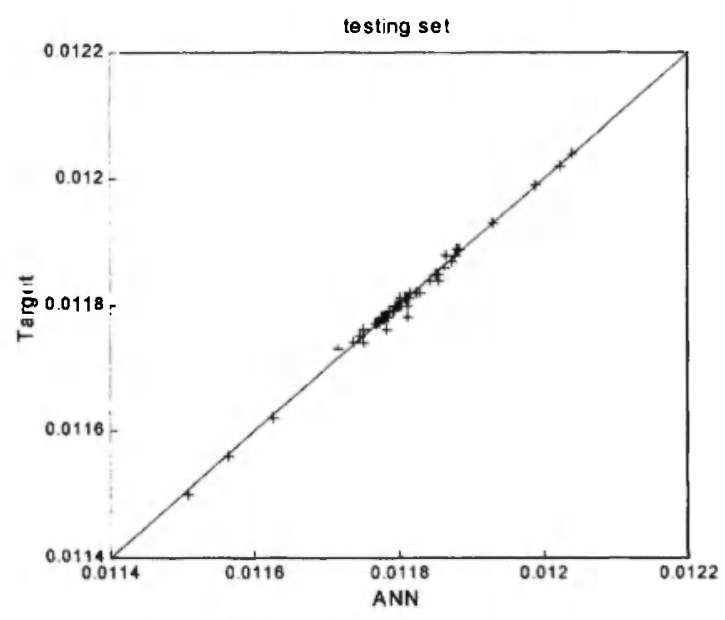

Fig. 10: Comparison of results for testing set

By changing the specimen width from $5 \mathrm{~mm}$ to 20 $\mathrm{mm}$, the effect of the coupon width on the uniformity of the strain field has been investigated (see Fig. II). It is clear that reducing gauge width reduces the deviation of the computed strains across the specimen. It may also be noted that a specimen with a width of less than $10 \mathrm{~mm}$ yields only a slight enhancement of the quality of the strain field. Furthermore, in considering physical testing limitations, a specimen width of less than $10 \mathrm{~mm}$ is not provide sufficient gauge width to accommodate a threeelement rectangular strain rosette. Therefore, the shape of the short coupon, selected by comparing strain variations across the centre of the gauge area and stress concentrations, is recommended to be straight sided with $10 \mathrm{~mm}$ wide. It may also be noted that even though only three potential geometry types have been assessed (reflecting fabrication constraints) the recommended straight-sided coupon geometry is consistent with ASTM guidance regarding highly uni-directional FRP for strength determination. Correlation between the FE and the ANN has been investigated by using the specimen width with $12.5 \mathrm{~mm}$. The uniformity of the strain field demonstrated excellent correlation with the ANN as shown in Fig. 11.

The effect of the gripping length on the strain distributions at the centre of the short coupon subjected to gripping length variations is reported. Changing the gripping length from $6 \mathrm{~mm}$ to $14 \mathrm{~mm}$ has an effect on the quality of the strain field (see Fig. 12). As expected, shorter gripping lengths tend to produce a more uniform strain distribution owing to the rapid dissipation of the 
clamping action of the jaws. Conversely, longer gripping length produced less uniform strain field as the gauge length decreased. Therefore, the uniformity of the strain field at the central region of the coupon is directly related to the gauge length. It can be seen from Fig. 12, the influence of a gripping length of less than $12 \mathrm{~mm}$ is not profound. Comparing the influences of geometry change and gripping length suggests that the effect of gripping length is less than the geometric variation. However, the gripping length becomes significant in the determination of the material strength. For practical reasons it is not feasible to use a gripping length shorter than $8 \mathrm{~mm}-10 \mathrm{~mm}$. The ANN results showed that the effect on the gripping length, with $12 \mathrm{~mm}$, showed excellent agreement with the $\mathrm{FE}$ results as plotted in Fig. 12.

Strain distributions at the centre of the short coupon subjected to length variations (see Fig. 13) show that for short lengths (less than $60 \mathrm{~mm}$ ) the computed strains in the central region of the coupon diverge from the analytical target. For specimens shorter than $60 \mathrm{~mm}$, the magnitude of possible error between the expected analytical and computed strains can be estimated. Then the factors are used to divide the measured strain in the evaluation of analytical strain from numerical or (more relevantly) experimental strain results for shorter lengths. It should be noted that these factors apply only to a straight-sided coupon with a width of $10 \mathrm{~mm}$ (equivalent aspect ratios also apply). The FE outcomes have been experienced using $35 \mathrm{~mm}$ length specimen and results found to be correlated with the FE results (see Fig.13).

The effect of specimen thickness on strain distribution is reported for thicknesses between $2 \mathrm{~mm}$ and $6 \mathrm{~mm}$ to assess the applicability of the recommended coupon geometry to other thicknesses. Plotting the magnitude of the longitudinal strain distribution across the width of the coupons indicated that an increase in thicknesses increases the uniformity of strain distribution at the centre (see Fig. 14).

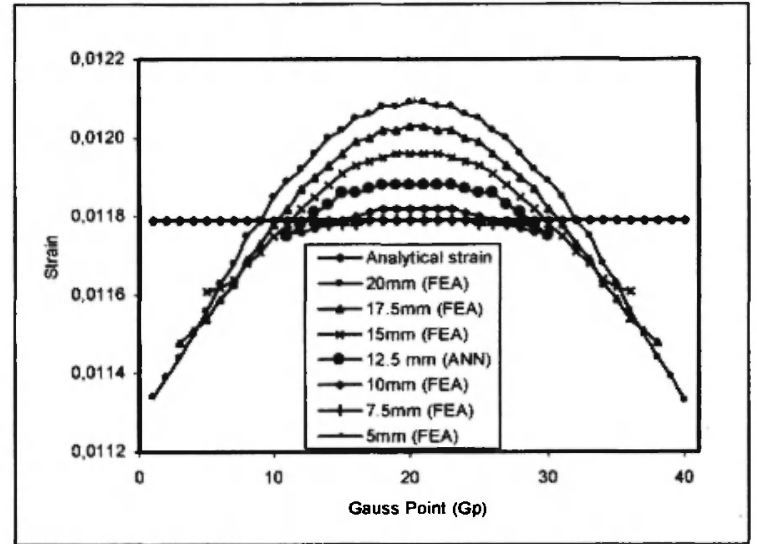

Fig. 11: Influence of coupon width on longitudinal strain distribution.

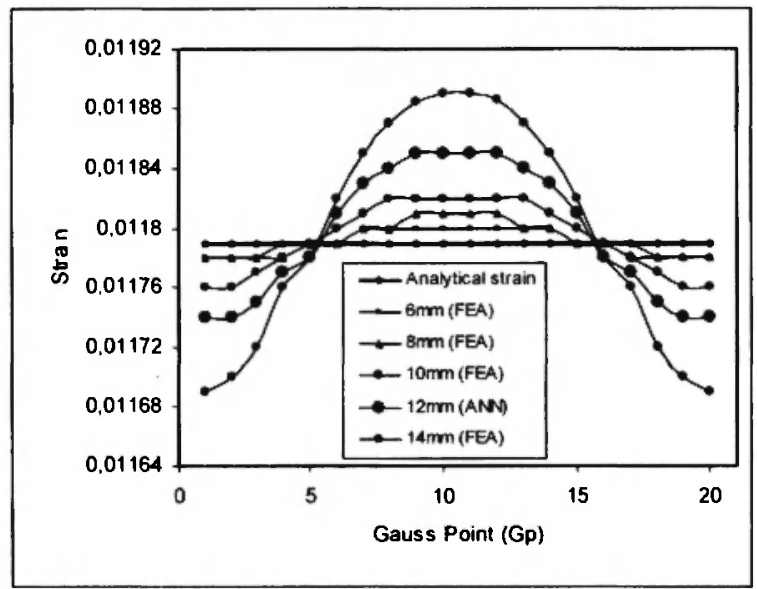

Fig. 12: Influence of gripping length on longitudinal strain distribution.

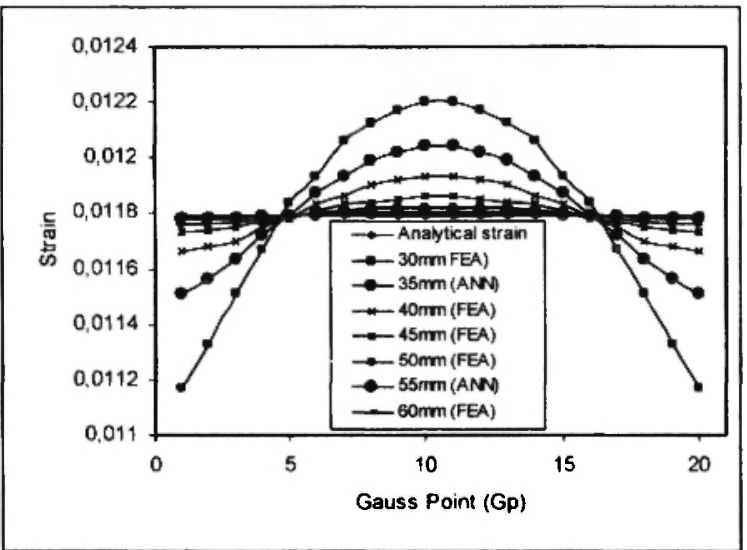

Fig. 13: Influence of coupon length on longitudinal strain distribution. 


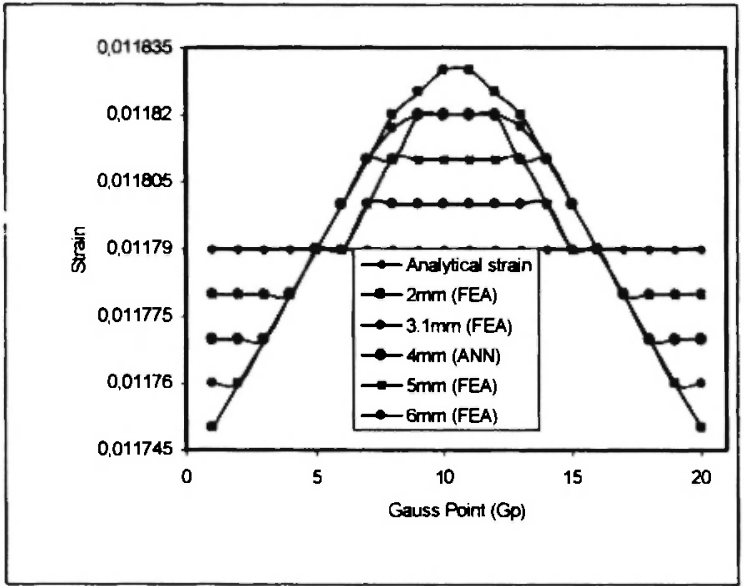

Fig. 14: Influence of coupon thickness on longitudinal strain distribution.

It has been demonstrated that the ANN based approach is highly successful to model the strain distribution of axially loaded short coupons for the determination of elastic material constants. Therefore, the training process of the ANN model takes quite long time. To overcome the time consuming training process SCGA, which is more powerful than CGA, has been used. Since the model trained, any problem can easily be modelled and analysed more rapidly when compared with the FE and classical solutions. A careful study of the results leads to observations of excellent agreement between the ANN predictions and the FE results. It appears to offer a means of dealing with many multivariety problems for which an exact analytical model does not exist or at least is very difficult and time consuming to develop. Therefore it is necessary to use a new algorithm which can overcome the time consuming problem and these difficulties.

\section{CONCLUSIONS}

The shape of a short tensile test coupon has been established using the finite element method and then the applicability of the ANN has been investigated. The FE model of the ASTM D3039 coupon has been established to validate the numerical coupon representation. Assuming a length of $50 \mathrm{~mm}$, the shape of this shortened coupon has been changed and assessed, leading to the specification of plan geometry. The following principal conclusions have been drawn:
- The FE method and the ANN have been successfully applied to model the strain distribution of axially loaded short coupons for the determination of elastic material constants. The ANN results are in good correlation with FE results and are examined against other results that are not used as part of the training data.

- The training process of the ANN model takes quite long time. To overcome this time consuming training process, the SCGA algorithm, which is more powerful than CGA algorithm, has been used. Since the model trained, any problem can easily be modelled and analysed more rapidly when compared with the finite element and classical solutions.

- Coupon length has been identified as an important geometrical parameter in the definition of a coupon test specimen. For specimens having a length of less than $60 \mathrm{~mm}$, the magnitude of the possible error between true and calculated strains has been estimated and an appropriate correction factor (acting on the measured strains) derived.

- Gripping length has been predicted not to significantly compromise the uniformity of the strain distribution in the central region of the short coupon. However, this parameter may become significant in the determination of the material strength.

- A straight-sided short coupon with $60 \mathrm{~mm}$ in length, $10 \mathrm{~mm}$ in width and $10 \mathrm{~mm}$ gripping lengths has been shown numerically to be an effective shape in cases where insufficient material is available to provide a standard specification coupon (ASTM D3039). The uniformity of the strain field has been used to measure effectiveness.

- The study indicated clearly that if the ANN system is combined with abounding data from constant reliable source, then the neural network based approach could effectively help to identify the short coupon shape for determination of material properties.

\section{REFERENCES}

1. J.F. Davalos, H.A. Salim, P. Qiao, R. Lopez-Anido and E.J. Barbero, "Analysis and design of pultruded FRP shapes under bending", Composites: Part B, 27B, 295-305 (1996). 
2. S.S. Sonti and E.J. Barbero, "Material characterisation of pultruded laminates and shapes", Journal of Reinforced Plastic and Composites, 15, 701-717 (1996).

3. S.M. Cron, A.N. Plazotto and R.S. Sandhu, "The improvement of end-boundary conditions for offaxis tension specimen use", Exp. Mechanics, 28, 14-19 (1988).

4. R.S. Sandhu and G.P. Sendeckyj, "On Design of Off-Axis specimen", Technical Report, AFWALTR-84-3098, Flight Dynamics Laboratory, Air Force Wright Aeronautical Laboratories, WrightPatterson Air Force Base, Ohio (1985).

5. M.H. Kural and D.L. Flaggs, "A finite element analysis composite tension specimens", Composites Technology and Research, 5, 11-17 (1983).

6. B.C. Foss, W.E. Wolfe and R.S. Sandhu, "The effect of tab orientation on the distribution of strains in composite specimens", Composite Materials: Testing and Design $\left(10^{\text {th }}\right.$ Vol.), ASTM STP 1120, Glenn C. Grimes, Ed., ASTM, Philadelphia, 103-113, 1992.

7. P.D. Gosling and M. Saribiyik, "Nonstandard tensile coupon for fibre reinforced plastic", $J$. Materials in Civil Engineering, 15 (2), 108-117 (2003).

8. B.D. Ripley, Pattern Recognition and Neural Networks, Cambridge Univ. Press, 1996.

9. S. Theodoridis and K. Koutroumbas, Pattern Recognition, London: Academic Press, 1999.

10. L. Fa-Long and U. Rolf, Applied Neural Networks for Signal Processing, Cambridge: Cambridge University Press, 1998.

11. L. Berke, S.N. Patnaik and P.L.N. Murthy, "Optimum design of aerospace structural components using neural networks", Computers \& Structures, 48 (6), 1001-1010 (1993).

12. H.T. Kang and C.J. Yoon, "Neural network approaches to aid simple truss design problems", Microcomputers in Civil Engineering, 9, 211-218 (1994).

13. G. Yagawa and H. Okuda, "Neural networks in computational mechanics", Arch. Comp. Meth. Eng., 3 (4), 435-512 (1996).

14. S.W. Liu, J.H. Huant, J.C. Sung and C.C. Lee,
"Detection of cracks using neural networks and computational mechanics", Comput. Methods Appl. Mech. Engrg. 191, 2831-2845 (2002).

15. E. Pabisek, M. Jakubek and Z. Waszczyszyn, "A fuzzy neural network for the analysis of experimental structural mechanics problems" In: L. Rutkowski and J. Kacprzyk (Eds.), Neural Networks and Soft Computing, Springer, PhysicaVerlag, Bertlin-Heidelberg, 2003; 772-777.

16. S.M.R. Emami, Y. Iwao and T. Harada, "A method for prediction of peak horizontal acceleration by artificial neural networks", Proceedings Elev., World Conference Earth. Engineering, paper No.1238, Rotterdam: Elsevier, 1996.

17. L. Hong-Nan and H. Lin-Sheng, "TLCD semiactive control of eccentric structures using neural networks", 15th ASCE Engineering Mechanics Conference, Columbia University, New York, NY, 2002.

18. K. Bani-Hani, J. Ghaboussi and S.P. Schneider, "Experimental study of identification and control of structures using neural network" Earthq. Eng. Struct. Dyn., 28, 995-1018 (1999).

19. J.A. Lee, D.P. Almond and B. Harris, "The use of neural networks for the prediction of fatigue lives of composite materials" Composites Part A: Applied Science and Manufacturing, 30, 11591169 (1999).

20. J. Kasperkiewics, "The application of ANNs in certain material-analysis problems", J. Material Processing Technology, 106, 74-79 (2000).

21. A.T. Goh, "Prediction of ultimate shear strength of deep beams using neural networks", $A C l$ Struct. Jour., 92 (1), 28-32 (1995).

22. H.N. Bar, M.R. Bhat and C.R.L. Murthy, "Identification of failure modes in GFRP using PVDF sensors: ANN approach", Composite Structures, [In Press, Corrected proof available online (2003)].

23. H.T. Kang and C.J. Yoon, "Neural network approaches to aid simple truss design problems" Microcomputers in Civil Engineering, 9, 211-218 (1994).

24. M. Saribiyik, "Analysis of a bonded connector for pultruded G.R.P. structural elements", Ph.D. Thesis, University of Newcastle, U.K., 2000. 
